\title{
Estudo do uso de Carbapenémicos em uma Unidade de Terapia Intensiva Adulta de um Hospital Público do Município de Vitória da Conquista-Bahia
}

\author{
Emerson Batista Santos $^{l}$; Karina Matos Pereira ${ }^{2}$
}

\begin{abstract}
Resumo: As organizações hospitalares estão inseridas num ambiente complexo que quando não funcionam de forma adequada gera problemas bastante gravosos. No setor saúde a unidade de terapia intensiva (UTI) é uma unidade muito difícil de ser lidada, já que abarca pacientes graves e casos onde precisa-se do máximo de cuidado possível e relacionado ao uso de medicamentos neste setor, a preocupação é latente pois há o risco de contaminação maior devido ao uso de antibióticos e no caso do uso do carbapenémicos não seria diferente. Assim será analisado neste estudo o uso deste tipo de antibiótico no trato de pacientes em UTI e analisar as nuances acerca do uso destas drogas de maior prescrição. Nota-se que foi analisado diversas nuances acerca do uso deste fármaco e tendo um resultado superior contra enterobactérias. Salienta-se aqui estudos que não consideram este medicamento o mais recomendado em unidade de terapia intensiva (UTI), por causa da resistência da Pseudomonas aeruginosa e da Acinetobacter baumannii, pois estas são bactérias que tem alta taxas de resistência. Como resultados as pesquisa, conforme as culturas feitas viu-se sensíveis aos carbapenémicos foram $124(78,48 \%)$ e resistentes aos carbapenémicos foram 34 (21,52 \%), e que o local mais incidência detectado foi a Secreção traqueal com 44,30\% sensível e 13,92\% resistente, e na urocultura com 12,02\% (sensíveis) e 0,63\% nas resistentes sendo que os microorganismos mais incidentes foram P. auruginosas, com 13,29\% nas sensíveis e 3,79\% nas resistentes, Acinetobacter baumani com 5,06\% (sensíveis) e 13,92\% (resistentes), Klebisiela pneumonie ssp com 14,55\% (sensíveis) e a $E$. coli com $10,75 \%$, nas sensíveis e não foram encontradas resistentes nestas duas últimas.
\end{abstract}

Palavras- chave: UTI. Uso. Carbapenémicos, Análise Clínica.

\section{A Study of the use of Carbapenems in an Adult Intensive Care Unit of a Public Hospital in Vitória da Conquista Municipality of Bahia State}

\begin{abstract}
Hospital organizations are embedded in a complex environment that does not function adequately for the rather onerous problems. In the health sector, the intensive care unit (ICU) is a very difficult unit to deal with, since it covers serious patients and cases where it is necessary to make the maximum possible care and related to the use of medicines in the sector, a concern Is latent because a risk of greater contamination in the use of antibiotics and in the case of the use of carbapenems would not be different. Thus analyzed in this study or use of this type of antibiotic without treatment of patients in the ICU and analyze as nuances on the use of drugs with higher prescription. It is noted that several nuances have been analyzed on the use of this drug and having a superior result against enterobacteria. Studies that do not consider this drug or more recommended in intensive care (ICU), because of the resistance of Pseudomonas aeruginosa and Acinetobacter baumannii, are bacteria that have high resistance rates. As a result of the research, the carbapenem effects of 124 (78.48\%) and resistant to carbapenems were $34(21.52 \%)$, 30\% sensitive and $13.92 \%$ resistant, and in uroculture with $12.02 \%$ ( ) And $0.63 \%$ in the resistant ones, with the most incident microorganisms being P. auruginosa, with $13.29 \%$ in the sensitive and $3.79 \%$ in the resistant, Acinetobacter baumani with 5.06\% (sensitive) and 13.92\% ( Resistant), Klebisiela pneumonie ssp with $14.55 \%$ (sensitive) and E. coli with $10.75 \%$, in the sensitive and were not found resistant in the latter two.
\end{abstract}

Key-words: ICU. Carbapenem Usage, Clinical Analysis.

\footnotetext{
${ }^{1}$ Acadêmico em Farmácia Generalista pela Faculdade Independente do Nordeste - FAINOR. Contato: emerson24horas@hotmail.com 2 Farmacêutica Bioquímica pela Universidade de Ribeirão Preto. Especialista em Farmacologia pela Universidade Federal de Lavras. Mestranda em Farmacologia Clínica pela Universidade Federal do Cariri. Docente da Faculdade Independente do Nordeste, departamento de saúde, Vitória da Conquista-Bahia. Docente da Universidade Estadual da Bahia, departamento de saúde, Vitória da Conquista-Bahia. Contato: karinamp09@gmail.com;
} 


\section{Introdução}

As organizações hospitalares estão inseridas num ambiente complexo e singular que as condiciona a um funcionamento inadequado diante da lógica, da acumulação lucrativa dos mercados, pois, inadequadamente a sua natureza, ambas as condições estão subordinadas a princípios éticos e legais que normatizaram o setor saúde e os políticos colocam os hospitais frente a uma diversidade de interesses divergentes a contemplar (CANINEU et al, 2006).

A unidade de terapia intensiva (UTI), por sua vez é uma unidade muito complexa, que está imersa em um sistema de monitorização perene que atende pacientes potencialmente graves que necessitem de um tratamento intensivo. Por ser um ambiente que requer maiores cuidados, máximo de eficiência, precisão, atenção e habilidade por parte dos profissionais junto ao paciente, por ser também um ambiente que suscita muitas fantasias e também maior contato com a morte do outro e consequentemente remetendo a sua própria finitude, sabe-se que nesta unidade o surgimento de problemas emocionais nestes profissionais é mais propício (LEÃO; CARPENTIER, 2002).

Segundo Nascimento; Trentini (2004) as UTIs possuem algumas características próprias, como a convivência diária dos profissionais e dos sujeitos doentes com as situações de risco; com ênfase na tecnologia para o atendimento biológico, com vistas a manter o ser humano vivo; enfrentando muitos problemas e tendo rotinas rígidas e inflexíveis, e a rapidez de ação no atendimento.

Tal fato denota certa preocupação acerca do uso de medicamentos neste setor, isto porque o risco de contaminação é maior e o uso de antibióticos é uma realidade constante, e como tal deve ser feita de forma adequada, como um suporte e atenção maior (SAMPAIO; MAGALHÃES, 2008).

No cenário da multirresistência, é importante frisar a questão dos Gram-negativos produtores de carbapenemases, vez que as infecções causadas por esses agentes são de importante morbimortalidade e seu tratamento é um dos maiores desafios no controle de infecção em Unidades de Terapia Intensiva (SILVA; SILVA JR, 2015).

O uso de Carbapenemicos na unidade de terapia intensiva de um hospital público da cidade de Vitória da Conquista-Ba é o tema deste estudo, vez que os antibióticos são medicamentos muito usuais nesta prática clínica fazendo toda a diferença no trato de pacientes 
em UTI e sendo uma das drogas de maior prescrição. Mas é relevante destacar os cuidados com o seu uso, vez que se não medicado como deve, na forma cautelosa acaba gerando um uso indiscriminado e inapropriado, que torna muitos patógenos resistentes e pode levar a um problema de graves sequelas (COCHRANE, 2002).

Por sua vez este trabalho trata sobre o uso dos carbapenemicos na unidade de terapia intensiva de um hospital público, trazendo as principais nuances a respeito do uso deste medicamento e quais as influências na área de infectologia e demonstrar assim os dados relacionados ao uso deste medicamento.

\section{Material e Métodos}

Esta pesquisa foi realizada através de um estudo de caráter epidemiológico que irá determinar a ocorrência e a distribuição de dados relativos ao uso de carbapenemicos (JEKEL; KATZ; ELMORE, 2005). Ainda na abordagem epidemiológica ela está caracterizada no estudo transversal de prevalência, no qual estuda analisa dados em um determinado local e tempo (BORDALO, 2006). O presente estudo possui natureza retrospectiva e descritiva, com abordagem quantitativa. Tal pesquisa foi realizada no Hospital Geral de Vitoria da Conquista, pautado no projeto que foi submetido a plataforma Brasil.

Para a elaboração deste trabalho, foram utilizados dados do projeto inicial descritos a cima, a partir de dados da população que o estudo atenderá consiste os pacientes internados nas UTI 1 do Hospital Geral de Vitória da Conquista em uso de carbapenemicos.

Tal pesquisa foi realizada através uma avaliação onde foram analisados os dados de prontuários de pacientes, e com as informações coletadas dos pacientes que fazem uso dos carbapenemicos.

Após a coleta dos dados, os mesmos foram transcritos e tabulados utilizando-se uma o programa Microsoft Office Excel®2010 contendo as variáveis em estudo, apresentados na forma de frequência e percentagem. Para a análise e interpretação dos dados da pesquisa será utilizado o método de análise quantitativa e qualitativa.

Tal estudo respeitou os aspectos éticos da pesquisa, sendo esta conduzida de acordo com a Resolução no 466/2012 da Comissão Nacional de Saúde, que reflete a pesquisa envolvendo 
seres humanos por ocasião da coleta de dados, sendo a coleta de dados iniciada somente após a autorização.

\section{Resultados e Discussâo}

A tabela a seguir é referente as culturas da amostra de culturas, onde $(78,48 \%)$ se mostraram sensíveis aos carbapenémicos e $(21,52 \%)$ resistente a este antibiótico em análise. Conforme tabela a seguir:

Tabela 1: Referente as culturas. Vitória da Conquista, Bahia, Brasil, 2017.

\begin{tabular}{l|c|c|c|c|}
\hline \multirow{2}{*}{ Dados } & \multicolumn{2}{|c|}{ Sensíveis aos carbapenémicos } & \multicolumn{2}{c}{ Resistentes aos carbapenémicos } \\
\hline Índices & $\mathrm{n}$ & $\%$ & $\mathrm{n}$ & $\%$ \\
\hline N (\%) & 124 & 78,48 & 34 & 21,52 \\
& & \multicolumn{4}{|c|}{158} \\
\hline Total: & \multicolumn{5}{|c}{} \\
\hline
\end{tabular}

Fonte: Dados da Pesquisa.

Da amostra acima, é preponderante destacar que das 158 (100\%) das culturas realizadas, 124 delas apresentaram-se sensíveis aos carbapenémicos, ou seja, 78,48\%. E os resistentes aos carbapenémicos foram $34(21,52 \%)$ componentes da amostra.

$\mathrm{Na}$ análise feita percebeu-se que o uso de fármacos aplicados na UTI visa melhorar doenças gravosas, curá-las e obter a sua máxima ausência de risco e falhas que possam prejudicar o paciente (NASCIMENTO; TRENTINI, 2009). E o uso de antibióticos, antimicrobianos é mais latente neste ambiente e deve ser feito dentro de condições adequadas, caso contrário passa a gerar problemas que prejudicará o paciente e o atendimento (SAMPAIO; MAGALHÃES, 2008).

Gales et al (2002) destaca resultados de carbapenémicos contra bacilos gram-negativos, especialmente contra amostras de P. aeruginosa e B. cepacia. Outros estudos têm demonstrado 
resultados semelhantes aos achados deste trabalho e confirmam a excelente atividade do meropenem contra bacilos gram-negativos $(8,23)$. Destacando neste estudo de foi sensível ao imipenem e resistente ao meropenem. Porém, mesmo testando apenas um dos carbapenêmicos, este estudo enfatiza que deve ser usado o carbapenêmico mais adequado para a situação clínica em questão.

Estudo feito por Melo et al (2012) destaca ainda que deve ser analisada a relação causaefeito, entre a exposição aos fármacos, principalmente no caso deste estudo analisando o, será associada a resistência bacteriana com estudos de natureza epidemiológica e clínica, analisando se há relação causal entre o uso destes e resistência bacteriana.

Nota-se que o uso indiscriminado dos medicamentos em pacientes é responsável pelo desenvolvimento de resistência de bactérias aos tópicos, e a importância do profissional de farmácia se dá pelo fato de que, dentro de um ambiente hospitalar, a seleção de medicamentos pela Comissão de Farmácia Terapêutica com bases em provas feitas que se pautam dentro da segurança e eficácia ajudam para o uso racional de medicamentos, evitando consequentemente que seja elevado o índice da virulência, atrasos na introdução da terapia adequada e menos opções de tratamento (PALCEVSKI et al, 2000).

Nota-se que o uso racional de antibióticos nas Unidades de Terapia Intensiva deve estar pautado em uma escolha terapêutica adequada com a indicação adaptada, considerando eficácia, segurança, conveniência para o paciente e custo; dose, administração e duração do tratamento apropriados; adesão ao tratamento pelo paciente (DIEFENTHAELER, 2007).

Burke (2003) destaca que deve ser feita a identificação de reações adversas e monitoramento de interações medicamentosas e Alissom (et al, 2008) destacam que por esse motivo o uso racional deve ser pautado na necessidade clínica, na dose e posologia corretas, por um período de tempo adequado, vez que se assim não for feito o uso de antibióticos ao invés de tratar e precaver uma infecção, eliminando os organismos patogênicos acabará piorando o quadro, gerando bactérias mais fortes e responsáveis por infecções difíceis de serem cuidadas.

Cruvinel et al (2011) destaca que esta bactéria é resistente ao ertapenem. E, é valido salientar que esta resistência está atrelada a capacidade de crescer testes de análise in vitro onde se analisa a concentração da droga. No entanto, o que se analisa nestes casos é de que a bactéria 
seja "resistente" a um determinado antimicrobiano no sangue, e por isso analisar quais destes fármacos são mais adequados para cada tipo de bactéria é importante (ANDRADE et al.,2006).

A tabela 2 abaixo refere-se a incidência de microorganismo, percebendo que as mais incidentes no quesito sensível ao carbapenemicos foram: a Klebisiela pneumonie esbl $(14,55 \%)$, P. auruginosas $(13,29 \%)$ e a E. Coli (10,75\%), salientando que da amostra de microorganismos resistentes obteve percentual maior na Acinetobacter baumani (12,92\%), sendo uma percentagem bastante alta quando comparadas a outros microorganismos.

Tabela 2: Referente à incidência de micro-organismos sensíveis e resistentes ao carbapenemicos. Vitória da Conquista, Bahia, Brasil, 2017.

\begin{tabular}{c|c|c|c|c}
\hline Microorganismos & \multicolumn{2}{|c|}{ Sensível } & \multicolumn{2}{c}{ Resistente } \\
\hline P. auruginosas & n & \% & n & \% \\
\hline P. auruginosas esbl & 21 & 13,29 & 06 & 3,79 \\
P. auruginosas R & 0 & 0 & 01 & 0,63 \\
Acinetobacter baumani & 0 & 0 & 03 & 1,89 \\
E. coli & 08 & 5,06 & 22 & 12,92 \\
E. coli esbl & 17 & 10,75 & 0 & 0 \\
Klebisiela pneumonie ssp pn & 03 & 1,91 & 0 & 0 \\
Klebisiela pneumonie esbl & 23 & 14,55 & 0 & 0 \\
Klebisiela pneumonie spp pn esbl & 08 & 5,06 & 0 & 0 \\
Klebisiela pneumonie & 15 & 9,49 & 0 & 0 \\
E. clocae esbl & 05 & 3,16 & 0 & 0 \\
E. clocae ibl & 08 & 5,06 & 01 & 0,63 \\
E. clocae & 05 & 3,16 & 0 & 0 \\
E. clocae mr & 01 & 0,63 & 0 & 0 \\
P. mirabilis ibl & 01 & 0,63 & 0 & 0 \\
P. mirabilis & 01 & 0,63 & 0 & 0 \\
M. morgani esbl & 01 & 0,63 & 0 & 0 \\
Providencia spp ibl & 02 & 1,26 & 0 & 0 \\
Proviencia spp esbl & 02 & 1,26 & 0 & 0 \\
P. vulgares esbl & 01 & 0,63 & 0 & 0 \\
S. maltophilia mr & 01 & 0,63 & 0 & 0 \\
S. saprophiticus & 0 & 0 & 01 & 0,63 \\
& 0 & 0 & 01 & 0,63 \\
& & & & \\
\hline
\end{tabular}

Fonte: Dados da Pesquisa. 
Com relação ao uso dos carbapenemicos, outros estudos atestaram a Klebisiela pneumonie esbl como mais incidente, sendo mais sensível a este fármaco, e neste mesmo estudo destacou ser eficiente no combate a E. coli (SEREFHANOGLU, 2009).

Martins e Barth (2013) e Almeida (2012) enfatizam a Acinetobacter baumannii como patógeno oportunista muito comum entre as mais incidentes infecções hospitalares, abarcando a bacteremia, meningite e infecção do trato urinário, o que demonstra muita cultura pela urina, sendo mais prevalente esta bactéria como agente de pneumonia nosocomial associada à ventilação mecânica, principalmente em UTI.

Casellas (2006) destaca que os carbapenêmicos mais usados estão o ertapenem, imipenem e meropenem e estes não devem ser muito usuais em unidade de terapia intensiva (UTI), por causa da resistência da Pseudomonas aeruginosa e da Acinetobacter baumannii, pois estas são bactérias que tem alta taxas de resistência a ele.

A tabela 3 refere-se ao local de cultura, e nota-se que a secreção traqueal foi a mais incidente tanto na sensibilidade $(44,3 \%)$ quanto na resistência $(13,92 \%)$, e a urocultura $(12,02 \%)$ para sensível. Conforme tabela 3, inframencionada destaca.

Viu-se que a maior frequência de exames aduz mais a secreção traqueal e urocultura como sítios de bactérias, conforme aduz Carvalho et al. (2005), tem alta incidência para cultura de secreção traqueal e ao perfil de resistência aos antimicrobianos. Miyaki (2005) atrela tal demanda a ventilação mecânica ou pelo fato de pacientes de Uti estarem a maioria intubados, por isso culturas deste tipo são comuns.

Os tipos de cultura demonstram que a maioria das infecções dos pacientes internados na UTI, advém de forma nosocomial, ou seja, do ambiente hospitalar, o que é agravado na UTI, já que nesta unidade há mais procedimentos mecânicos, necessitando de alerta para a lavagem de mãos de todos que frequentam este ambiente e controle de infecção, com a desinfecção e esterilização dos instrumentos usuais como cateter, drenos, respiradores mecânicos, sondas e outros. Tal situação é fator de risco para infecção já que as ações não são cautelosas como deveriam ser e isso gera uma relação causa-efeito que pode incidir em resistência bacteriana (MELO et al., 2012). 
Tabela 3: Referente ao Sítio. Vitória da Conquista, Bahia, Brasil, 2017.

\begin{tabular}{c|c|c|c|c}
\hline Micro-organismos & \multicolumn{2}{|c|}{ Sensível } & \multicolumn{2}{c}{ Resistente } \\
\hline Sec Traqueal & N & \% & n & \% \\
\hline Urocultura & 70 & 44,3 & 22 & 13,92 \\
Sec Ferida & 19 & 12,02 & 01 & 0,63 \\
Sec Sacral & 09 & 5,69 & 0 & 0 \\
Ponta de cateter & 04 & 2,53 & 02 & 1,26 \\
Sec Geral & 05 & 3,16 & 03 & 1,89 \\
Sec Uretral & 05 & 3,16 & 02 & 1,26 \\
Hemocultura & 01 & 0,63 & 0 & 0 \\
Cult Fraquemen & 02 & 1,26 & 0 & 0 \\
Sec Absômen & 02 & 1,26 & 0 & 0 \\
MSD & 01 & 0,63 & 0 & 0 \\
Liquor & 0 & 0 & 01 & 0,63 \\
Sec Ouvido & 0 & 0 & 01 & 0,63 \\
Sec Toraxica & 01 & 0,63 & 0 & 0 \\
MIE & 01 & 0,63 & 0 & 0 \\
Sec Escara & 01 & 0,63 & 0 & 0 \\
Sec Flanco & 01 & 0,63 & 0 & 0 \\
Sec Gastrica & 01 & 0,63 & 0 & 0 \\
Sec Abcesso & 01 & 0,63 & 0 & 0 \\
& 01 & 0,63 & 0 & 0 \\
Total: & & & & 21,52 \\
\hline
\end{tabular}

Fonte: Dados da Pesquisa.

Desta forma, é dever das comissões como a CCIH, Comissões Estaduais de Controle de Infecção Hospitalar e Comissões Municipais de Controle de Infecções Hospitalares implementar ações em prol da manutenção, efetivação e fiscalização de ações de controle de infecção hospitalar relacionada ao uso de fármacos e resistência bacteriana (ANVISA, 2007). 


\section{Conclusão}

Conclui-se que os carbapenêmicos são muito usados em UTI por ter um amplo espectro de atividade que abrange bactérias gram-positivas, gram-negativas e anaeróbias, tendo um resultado superior contra enterobactérias. Mas é interessante analisar que este fármacos carbapenêmicos, devem ser ministrados com muita cautela, vez que os carbapenemicos, não devem ser o mais recomendado em unidade de terapia intensiva (UTI), por causa da resistência da Acinetobacter baumannii, pois estas são bactérias que tem alta taxas de resistência, conforme foi atestado neste estudo.

\section{Referências}

ALMEIDA, Lais Pinto de et al. Ertapebem disk performance to predict Klebsiella pneumoniae carbapenemase produced by Gram-negative bacilli isolated in a São Paulo city public hospital. Einstein (São Paulo), São Paulo, v. 10, n. 4, p. 439-441, Dec. 2012. Disponível em: $<$ http://www.scielo.br/scielo.php?script= $45082012000400008 \& \operatorname{lng}=$ en\&nrm=iso>. Acesso em 10 abr 2017.

ANDRADE, Denise de; LEOPOLDO, Vanessa Cristina; HAAS, Vanderlei José. Ocorrência de bactérias multiresistentes em um centro de Terapia Intensiva de Hospital brasileiro de emergências. Rev. bras. ter. intensiva, São Paulo, v. 18, n. 1, p. 27-33, Mar. $2006 .$.

ANVISA. Agência Nacional de Vigilância Sanitária. Investigação e Controle de Bactérias Multirresistentes 2007. Disponível em: <http://www.professores.uff.br /jorge/manual\% 20 controle bacterias.pdf $>02$ fev 2017.

BARROS. Lívia Moreira; BENTO. John Nilbérick de Castro; CAETANO. Joselany Áfio; MOREIRA. Rosa Aparecida Nogueira; PEREIRA. Francisco Gilberto Fernandes; FROTA. Natasha Marques; ARAUJO. Thiago Moura de; SOARES. Enedina. Prevalência de micro-organismo e sensibilidade antimicrobiana de infecções hospitalares em unidade de terapia intensiva de hospital público no Brasil. Rev Ciênc Farm Básica Aplicada. 2012. Disponível em: <servbib.fcfar.unesp.br/seer/ index.php/Cien_Farm/article/viewFile/2211/126> Acesso em 17 abr 2017.

BORDALO, A. A. Estudo transversal e/ou longitudinal. Belém- PA: Revista Paraense de Medicina, 2006.

BRASIL. Lei no 5.991, de 17 de dezembro de 1973. Dispõe sobre o Controle Sanitário do Comércio de Drogas, Medicamentos, Insumos Farmacêuticos e Correlatos, e dá outras Providências. Disponível em: < http://www.planalto.gov.br/ccivil_ 03/leis/L5991.htm> Acesso em 30 mar 20175. 
BURKE, R.F. Infecção controle um problema para a segurança do paciente, Revista Ciência e Saude. 2003.

CANINEU. Rafael;GUIMARÃES. Hélio Penna, LOPES. Renato Delascio;VENDRAMEL. Letícia Sandre, JUNIOR. Max Artur da Fonseca; LOPES. Antonio Carlos. Iatrogenia em Medicina Intensiva, Rev. bras. ter. intensiva vol.18 no.1 São Paulo Jan./Mar. 2006. Disponível em: < http://www.scielo.br/scielo.php?pid=S0103-507X2006000100015 \&script=sc i art text $>$ Acesso em 09 mar 2017.

CARVALHO, Cid E. et al .Monitoramento microbiológico seqüencial da secreção traqueal em pacientes intubados internados em unidade de terapia intensiva pediátrica. J. Pediatr. (Rio J.), Porto Alegre, v. 81, n. 1, p. 29-33, Feb. 2005.

CASELLAS. José María. Resistência bacteriana na unidade de terapia intensiva. Critical Connections. 2006.

COCHRANE. Ertapenem - Canadian Coordinating Office for Health Technology Assessment. Ertapenem. Ottawa: Canadian Coordinating Office for Health Technology Assessment (CCOHTA) 2002.

DIEFENTHAELER, Helissara S. Avaliação da prescrição de antimicrobianos de uso restrito em um hospital universitário de Passo Fundo/RS. Dissertação (Mestrado),. Universidade Federal do Rio Grande do Sul. Faculdade de Farmácia. Programa de Pós-Graduação em Ciências Farmacêuticas. 2007.

GALES, Ana C. et al . Comparação das atividades antimicrobianas de meropenem e imipenem/cilastatina: o laboratório necessita testar rotineiramente os dois antimicrobianos?. J. Bras. Patol. Med. Lab., Rio de Janeiro, v. 38, n. 1, p. 13-20, Jan. 2002.

JEKEL; J.F. KATZ; D. L. ELMORE; J.G. Epidemiologia, Bioestatística e Medicina Preventiva. $2^{\text {a }}$ Ed. São Paulo: Artmed, 2005.

LEÃO, Luciene Soares Carneiro; CARPENTIERI, Mariana. Auto-produção de Stress e o Stress no Ambiente de UTI. 2002. Disponível em: <http://74.125.93.132/search?q=cache:YID1JB6AE8J:www.cphd.com.br/trabalhos/

cphd_11022006112316.doc+qualidade+de+vida+nos+profissionais+trabalham+na+UTI\&cd=38\&hl=p t-BR\&ct=clnk\&gl=br>. Acesso em: 27 fev 2017.

MARTINS, Andreza Francisco; BARTH, Afonso Luís . Acinetobacter multirresistente - um desafio para a saúde pública, Scientia Medica (Porto Alegre), 2013; volume 23, número 1, p. 56-62..

MELO, Viviane Vieira; DUARTE, Izabel de Paula; SOARES, Amanda Queiroz. Guia de antimicrobianos., 1.ed. Goiânia, 2012.

MIYAKI, Mitsuru. Monitoramento microbiológico seqüencial da secreção traqueal em pacientes intubados internados em unidade de terapia intensiva pediátrica. J. Pediatr. (Rio J.), Porto Alegre, v. 81, n. 1, p. 3-4, Feb. 2005.

NASCIMENTO, E.R.P.; TRENTINI, M. O cuidado na unidade de terapia intensiva (UTI): Teoria humanística de Paterson e Zderad. Revista Latino-americana de Enfermagem, 2009. Disponível em: $<$ http://www.scielo.br/pdf/rl ae/v12n2/v12n2a15.pdf $>$. Acesso em: 8 mar 2017. 
Id on Line Revista Multidisciplinar e de Psicoloqia

Id on Line Multidisciplinary and Psycology Journal

PALCEVSKI. V; MOROVIC. M, PALCEVSKI.G. Utilização de antibióticos no hospital universitário após a introdução de uma política de antibiótico., Rev. bras. ter. intensiva. 2000.

SAMPAIO, A.P.; MAGALHÃES, M.S.; A humanização da assistência na Unidade de Terapia Intensiva na visão dos usuários. RBPS. 2008.

SEREFHANOGLU, Kivanc et al . Bloodstream infections caused by ESBL-producing E. Coli and K. pneumoniae: risk factors for multidrug-resistance. Braz J Infect Dis, Salvador, v. 13, n. 6, p. 403407, Dec. 2009.

SILVA, Camila Delfino Ribeiro da; SILVA JUNIOR, Moacyr. Estratégias para uso adequado de antibioticoterapia em unidade de terapia intensiva. Einstein (São Paulo), São Paulo, v. 13, n. 3, p. 448-453, Sept. 2015.

\section{Como citar este artigo (Formato ABNT):}

SANTOS, EMERSON B.; PEREIRA, KARINA M. Estudo do uso de Carbapenémicos em uma Unidade de Terapia Intensiva Adulta de um Hospital Público do Município de Vitória da Conquista-Bahia. Id on Line Revista Multidisciplinar e de Psicologia, Maio de 2017, vol.11, n.35, p.188-198. ISSN: 1981-1179.

Recebido: 23.05.2017

Aceito: 23.05 .2017 